\title{
LAS ECUACIONES DIFERENCIALES ORDINARIAS EN UN CONTEXTO REALISTA
}

\author{
Juan Nápoles Valdés \\ jnapoles@exa.unne.edu.ar \\ https://orcid.org/0000-0003-2470-1090 \\ Universidad del Nordeste, Corrientes, Argentina \\ Osvaldo Jesús Rojas Velázquez \\ orojasv69@uan.edu.co \\ https://orcid.org/0000-0001-9015-2400 \\ Universidad Antonio Nariño, Bogotá, Colombia
}

Recibido: 15/04/2020 Aceptado: 23/mayo/2020

\begin{abstract}
Resumen
Indudablemente las ciencias matemáticas, así como el ejercicio de su enseñanza, a lo largo de toda su historia, siempre han tenido como principal medio y fin la resolución de problemas matemáticos. Halmos (1980) no puede ser más elocuente al respecto, cuando afirma que los problemas son "el corazón de la Matemática”. Resolver un problema debe servir no sólo para un simple entrenamiento intelectual, sino también para un efecto educativo formativo, para ilustrar lo anterior con suficiente claridad, hemos tomado un tema no demasiado heterogéneo, las ecuaciones diferenciales ordinarias. Presentamos un problema muy actual, un modelo de difusión de una epidemia, discutimos su solución y enfoques y hacemos algunas reflexiones educativas, desde las posiciones de la Matemática Realista.
\end{abstract}

Palabras clave: Matemática Realista, Ecuaciones Diferenciales, Modelación.

\section{AS EQUAÇÕES DIFERENCIAIS ORDINÁRIAS EM UM CONTEXTO REALISTA}

\section{Resumo}

Indubitavelmente, as ciências matemáticas, bem como o exercício de seu ensino, ao longo de sua história, sempre tiveram como principal meio e finalidade a resolução de problemas matemáticos. Halmos (1980) não poderia ser mais eloquente a esse respeito, quando afirma que os problemas são "o coração da matemática". A resolução de um problema deve servir não apenas para um treinamento intelectual simples, mas também para um efeito educacional formativo, para ilustrar o exposto com clareza suficiente, adotamos um tópico não muito heterogêneo, equações diferenciais ordinárias. Apresentamos um problema muito atual, um modelo para a disseminação de uma epidemia, discutimos sua solução e abordagens, e fazemos algumas reflexões educacionais, a partir das posições da Matemática Realista.

Palavras chaves: Matemática Realista, Equações Diferenciais, Modelagem 


\title{
ORDINARY DIFFERENTIAL EQUATIONS IN A REALISTIC CONTEXT
}

\begin{abstract}
Undoubtedly, the mathematical sciences, as well as the exercise of their teaching, throughout their history, have always had as their main means and end the resolution of mathematical problems. Halmos (1980) could not be more eloquent in this regard, when he states that problems are "the heart of mathematics". Solving a problem should serve not only for a simple intellectual training, but also for a formative educational effect, to illustrate the above with sufficient clarity, we have taken a not too heterogeneous topic, ordinary differential equations. We present a very current problem, a model for spreading an epidemic, discuss its solution and approaches, and make some educational reflections, from the positions of Realistic Mathematics.
\end{abstract}

Keywords: Realistic Mathematics, Differential Equations, Modeling

\section{Preliminares}

Uno de los fundamentos de la actual reforma de la enseñanza de la matemática es el concepto del que se parte respecto a la naturaleza del conocimiento matemático. La perspectiva histórica permite mostrar, entre otras cosas, que la matemática es un conjunto de conocimientos en evolución continua y que en esta evolución desempeña a menudo un papel de primer orden su interrelación con otros conocimientos y la necesidad de resolver determinados problemas prácticos.

En esta dirección, cabe señalar que el marco didáctico-metodológico, en el que está enmarcado nuestro trabajo, es el siguiente:

1. Concebir de manera dinámica a la matemática, lo que se expresa en la célebre frase de Philip E. Jourdain, en la introducción a su texto "La naturaleza de la matemática", cuando al declarar el objetivo central apuntaba: "Espero que conseguiré mostrar que el proceso del descubrimiento matemático es algo vivo y en desarrollo" (Jourdain, 1976) ${ }^{1}$. Esta concepción se refleja en una enseñanza basada en la resolución de problemas, tanto para el desarrollo de diversas habilidades lógicas de los alumnos, como para aclarar cuáles de aquellos hechos fueron los que motivaron el surgimiento de un concepto y por qué, cuál era el marco de rigor en aquel entonces, cuál la metodología, las concepciones y su influencia en el proceso de enseñanza-aprendizaje hoy.

2. Aceptar el triple significado de los objetos matemáticos: institucional, personal y temporal (Godino \& Batanero, 1994; Nápoles, 1997)².

\footnotetext{
${ }^{1}$ Philip E. B. Jourdain, matemático francés (1879-1919)

${ }^{2}$ El conocimiento se produce con continuidad temporal y no sólo en el ámbito reconocido institucionalmente para ese fin, se produce en todos los ámbitos de la vida humana. Los distintos conocimientos que se producen se pueden parcelar para su análisis y calificar a cada una de las facetas separadas con un nombre diferente, sin embargo en el sujeto que conoce tal separación es imposible de modo que la actividad matemática ha de tener en cuenta tal diversidad de fuentes de conocimiento así como los condicionantes que tiene el conocimiento matemático inmerso
} 
3. Distinguir entre una argumentación, una prueba y una demostración, y la necesaria dosificación de éstas en el currículo escolar, así como las discusiones en torno a las concepciones clásicas sobre la demostración matemática y el marco de rigor de las mismas $^{3}$. Así, se prefieren en ocasiones pruebas que expliquen (incluso en la labor docente, a veces más que una demostración es necesaria y útil una “discusión"), en vez de pruebas que sólo "prueben". Han adquirido relevancia, en los últimos tiempos incluso, las llamadas pruebas sin palabras, donde las representaciones geométricas vendrían a jugar el papel de las explicaciones necesarias.

4. Que existen diferencias cualitativas entre el funcionamiento académico (a nivel de investigación, como "saber sabio") de un determinado conocimiento y el funcionamiento didáctico del mismo ya que, por diversas causas, los usos y connotaciones de las nociones Matemáticas tratadas en las instituciones de enseñanza son necesariamente restringidas.

5. Que a los estudiantes es conveniente ofrecerles situaciones problemáticas que pueden imaginar, realistas. En la Educación Matemática Realista, los problemas presentados a los estudiantes pueden venir del mundo real concreto, pero también ser elaborados (pseudo-reales o completamente ficticios) o del mundo formal de las Matemáticas, siempre y cuando estos problemas sean concebidos como reales por los estudiantes.

Partamos de una cuestión central, este enfoque ¿qué ventajas nos ofrece? Entre otras podemos destacar las siguientes:

a. Aprende nuevas matemáticas participando en actividades matemáticas.

b. Modifican sus propias concepciones y creencias sobre la naturaleza de las matemáticas.

c. Permite construir modelos de pensamiento estudiantil y estudiar sus modos de actuación.

d. Aprenden "nuevas" matemáticas.

¿Por qué usar ecuaciones diferenciales?

a. Es un curso importante para una gran variedad de especialidades.

b. Es un área en la que se ha llevado a cabo poca investigación educativa.

c. Los cursos siguen un enfoque tradicional: organizado principalmente en torno a métodos analíticos de solución.

d. Es un curso organizado alrededor de la modelación, con métodos gráficos, numéricos y cualitativos.

e. Tiene fuertes conexiones con el cálculo y el álgebra lineal.

en el potente conocimiento cultural. Ambos planos de análisis de dentro y de fuera de las instituciones escolares, creemos que pueden ser válidos para aportar luz sobre los procesos cognitivos.

${ }^{3}$ Sucintamente podemos decir, que una argumentación es la acción de hacerle saber algo a alguien, puede que uno mismo, que una prueba es un tipo especial de argumentación que incorpora un valor epistémico verdadero y que demostración es una prueba lógicamente concluyente. 


\section{Referentes sobre la modelación y las EDO}

Para entender los diferentes modelos que se usan para representar las dinámicas poblacionales, empecemos por la ecuación general del crecimiento exponencial $\frac{d P}{d t}=k P$ donde P es el tamaño de la población, k es la tasa de aumento per cápita y t es el tiempo. (Figura 1).

Figura 1. Gráfica de la Ecuación General del Crecimiento Exponencial

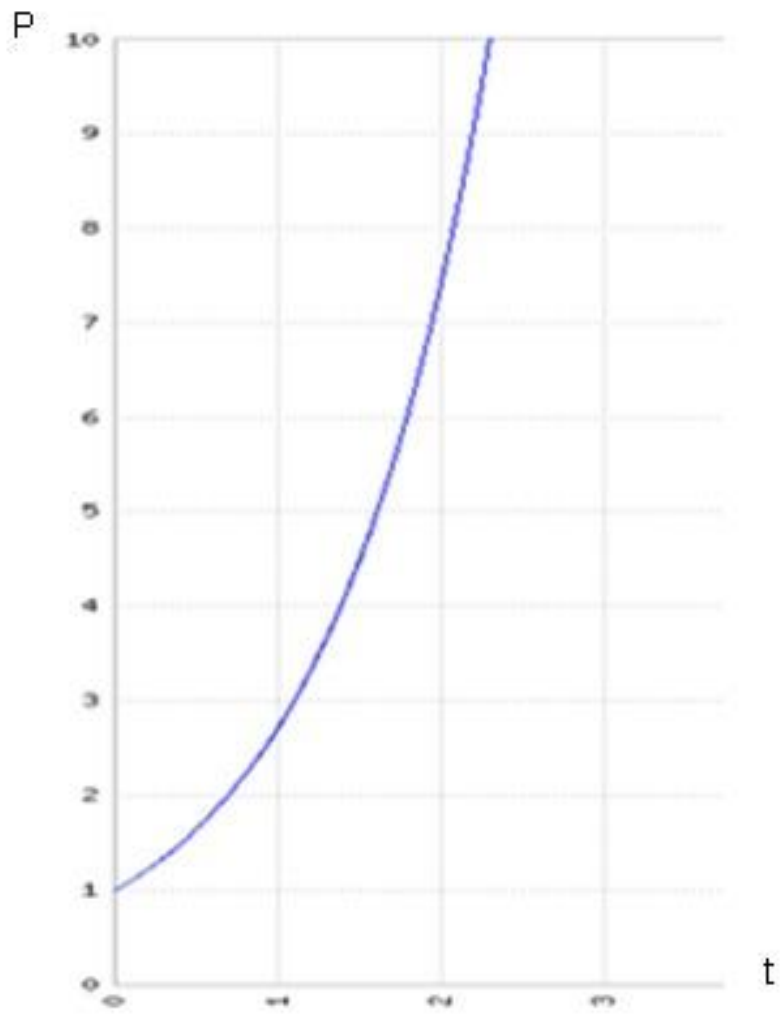

Las bacterias cultivadas en el laboratorio son un excelente ejemplo de crecimiento exponencial. En el crecimiento exponencial, la tasa de crecimiento de la población aumenta con el tiempo, en proporción con el tamaño de la población, $P(t)=P_{0} e^{k t}$, aquí $\mathrm{P}_{0}$ es la población inicial y $\mathrm{k}$ es el factor de proporcionalidad, derivada de las tasas de crecimiento de la población, en unidad de tiempo.

El concepto fundamental del crecimiento exponencial es que la tasa de crecimiento poblacional, el número de organismos que se añade en cada generación, aumenta al mismo tiempo que la población se hace más grande. Los resultados pueden ser dramáticos: ¡la población puede crecer sin límite! Algo que los propios estudiantes pueden obtener desde el gráfico anterior. 
Pasemos a una situación más real, en esa población hay muertes, es decir, no crece indefinidamente, ahí pasamos a una nueva situación: la tasa de crecimiento poblacional disminuye a medida que la población alcanza su límite máximo, entonces tenemos un crecimiento logístico. En este caso escribiremos, simplificadamente $P(t)=\frac{P_{0} e^{k t}}{1+P_{0} e^{k t}}$.

Lo sencillo es que el modelo es una ecuación diferencial de variables separables, bastante fácil de resolver $\frac{d P}{d t}=P(1-P)$, la gráfica en este caso es el siguiente se muestra en la Figura 2.

Figura 2: Gráfica de un Crecimiento Logístico:

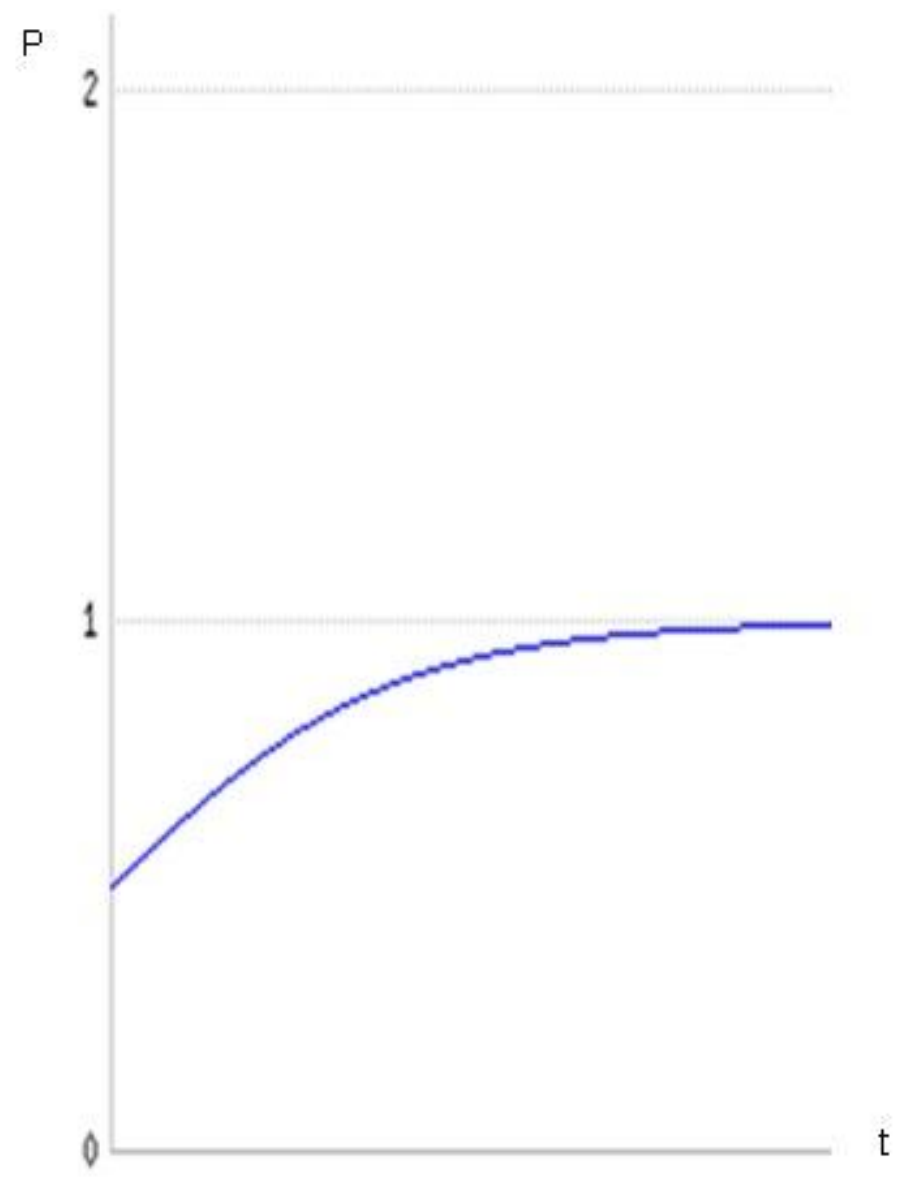

Aquí 1 representa la carga máxima del universo.

Si nos detuviéramos aquí, tendríamos solo la mitad del camino recorrido, podemos dar el salto y proponer un problema más teórico.

Resolver la ecuación diferencial $\frac{d x}{d t}=\left(x^{2}-1\right)$, cuya representación gráfica se muestra en la Figura 3. 
Figura 3. Trayectorias de la ecuación diferencial $\frac{d x}{d t}=\left(x^{2}-1\right)$

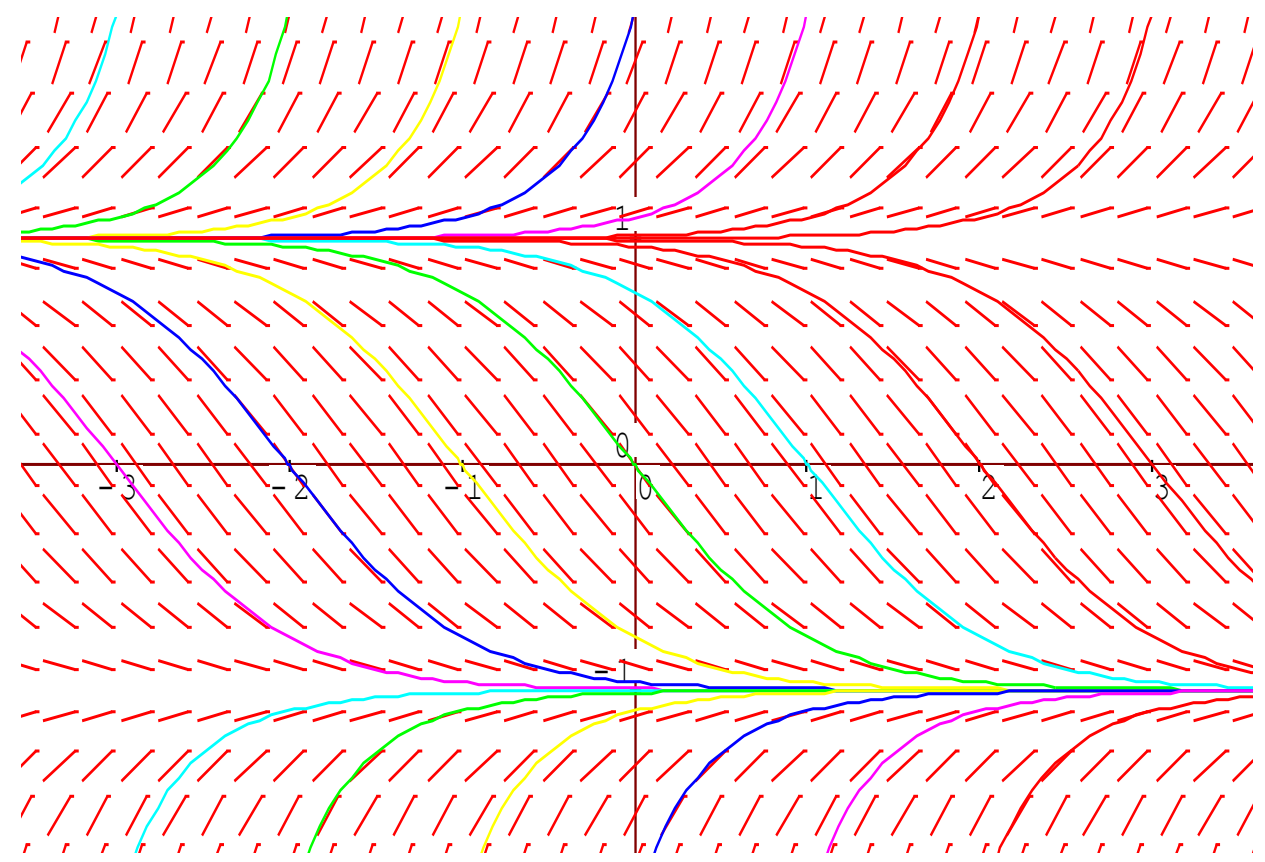

Si analizamos el marco algebraico, su solución es muy elemental, pues es una ecuación en variables separables y resoluble en cuadraturas, cuya solución se puede expresar por $x=\frac{1+c e^{2 t}}{1-c e^{2 t}}$, es claro que, esta expresión a los estudiantes no les dice mucho sobre el comportamiento de las soluciones: así declaran que dicho límite es -1. Aún teniendo el comportamiento gráfico (ver figura siguiente), es necesario realizar el análisis de cuándo el límite es -1 , ocasión propicia para revelar la necesidad de tener en cuenta las condiciones iniciales y el análisis general del problema. De nuevo recalcamos la necesidad de la integración de los diferentes acercamientos para completar el análisis.

\section{Referentes teóricos sobre las TIC, la visualización matemática y la Educación Matemática Realista.}

Para lograr un robusto proceso de enseñanza y aprendizaje de las ecuaciones diferenciales ordinarias, que permita que los estudiantes sean capaces de resolver problemas intramatemático y extramatemático, se hace necesario considerar en este proceso los referentes sobre: las TIC, visualización Matemática, Educación Matemática Realista. A continuación, se explican cada uno de estas teorías. 
Referentes sobre las TIC en el proceso de enseñanza y aprendizaje de la Matemática.

Las tecnologías de la información y la comunicación (TIC) han tenido una gran influencia en la enseñanza de las diferentes áreas del conocimiento en los últimos años en los diferentes niveles educativos. Concerniente al uso de las TIC en el proceso de enseñanza y aprendizaje de la Matemática, Santos, Moreno \& Camacho (2016, p. 828) plantean que: “... el uso de la tecnología digital ofrece a los docentes y estudiantes la oportunidad de ampliar y profundizar las formas de razonamiento sobre las estrategias matemáticas involucradas en la resolución de problemas” (Santos, Moreno \& Camacho, 2016).

Estos autores consideran además que representar y explorar tareas matemáticas mediadas por tecnologías digitales presenta nuevos desafíos para los docentes. En este sentido, es un campo que, pese a su rápida expansión, ofrece un sinnúmero de posibilidades novedosas de aplicación.

Integrar las TIC, específicamente software de GeoGebra, Matemática, entre otros, en las clases de ecuaciones diferenciales ordinaria contribuye a su enseñanza y aprendizaje, pues le brinda al docente de Matemáticas la oportunidad de crear ambientes de aprendizajes enriquecidos para que los estudiantes perciban esta área del conocimiento como una ciencia experimental. En este proceso los estudiantes se dotan de estrategias que le permiten aplicar las TIC al proceso de resolución de problemas intra y extramatemáticos.

Fundamentos de la visualización Matemática.

Arcavi (2003), Hershkowitz (1990), Zimmermann y Cunningham (1991), entre otros, han investigado sobre la visualización matemática. Según Arcavi (2003) la visualización es:

[...] la capacidad, el proceso y el producto de creación, interpretación, uso y reflexión sobre fotos, imágenes, diagramas, en nuestra mente, sobre el papel o con herramientas tecnológicas, con el propósito de representar y comunicar información sobre el pensamiento y desarrollo de ideas previamente desconocidas y avanzar en la comprensión (Arcavi, 2003, p. 217).

El proceso de visualización constituye un recurso para la comprensión de los conceptos matemáticos y para comunicar ideas matemáticas (Arcavi 2003). Estas ideas se consideran fundamentales en esta investigación. Para De Guzmán (1996) “... la visualización aparece como algo profundamente natural tanto en el nacimiento del pensamiento matemático como en 
el descubrimiento de nuevas relaciones entre los objetos matemáticos, y también, naturalmente, en la transmisión y comunicación propias del quehacer matemático (Guzmán, 1996, p. 3)".

En este trabajo se asume la visualización como un proceso que le permite al estudiante establecer relaciones entre los elementos propios de un objeto de ecuaciones diferenciales ordinarias y sus propiedades, para contribuir a la resolución de problemas intramatemático y extramatemático de su contexto.

Referentes sobre la Educación Matemática Realista.

La Educación Matemática Realista (EMR) se fundamenta en "herramientas conceptuales para una teoría de la educación matemática (Freudenthal, 1973, p. 31)”. Es una teoría que posibilita un proceso de enseñanza aprendizaje de la matemática escolar a partir de situaciones de la vida cotidiana o problemas extramatematicos de un contexto significativo para el estudiante.

En la EMR la matematización es una actividad estructurada y organizada que deviene del quehacer matemático, la cual está al alcance de todos los seres humanos. Es actividad es definida por Freudenthal (ídem, p. 44), como: "Matematizar es organizar la realidad con medios matemáticos...incluida la matemática misma”.

Según Freudenthal (ibídem, pp 35, 36), el proceso de matematización involucra:

1. Reconocer características esenciales.

2. Descubrir características comunes.

3. Ejemplificar ideas generales.

4. Enfrentar situaciones problemáticas.

5. La irrupción repentina de nuevos objetos mentales y operaciones.

6. Buscar atajos y abreviar estrategias y simbolizaciones.

7. Reflexionar acerca de la actividad matematizadora, considerando los fenómenos en cuestión desde diferentes perspectivas.

Estos elementos que involucra la matematización se es necesario tenerlos presente para el proceso de enseñanza aprendizaje de la resolución de problemas sobre ecuaciones diferenciales ordinarias. Pues se trata de resolver situaciones o problemas con contexto real y a la vez de interés para los estudiantes, como puede ser el caso del Coronavirus. 


\section{El caso del Coronavirus.}

Un problema muy actual es la pandemia del COVID-19, por ello puede ser utilizado en diferentes formas, en particular desde el punto de vista educativo.

Para ello utilizaremos el Modelo SIR (Kermack \& McKendrick, 1927; Uribarri, Rodríguez \& Cervantes, 2013-2014), cuyas siglas provienen de considerar tres clases de personas: el número de los individuos susceptibles $\mathrm{S}(\mathrm{t})$, es decir aquellos que pueden contagiarse; los infecciosos I(t), los que al estar enfermos pueden transmitir la enfermedad; los recuperados $\mathrm{R}(\mathrm{t})$, aquellos que después de enfermarse y aliviarse quedan en un estado de inmunidad o bien están aislados en algún sitio (como un hospital) o fallecieron. Este modelo tiene varias restricciones:

a. Una sola infección es la responsable de ocasionar un proceso infeccioso en el huésped.

b. El desenlace de la enfermedad es la muerte o la inmunidad completa.

c. La tasa de contagio es proporcional al número de enfermos.

d. Todos los individuos sanos son susceptibles.

e. La población es cerrada, en la práctica esto es prácticamente imposible como se ha visto en la pandemia que nos ocupa.

f. El periodo de incubación es muy corto, en particular se usa 14 días en este caso.

g. El individuo susceptible se enferma y puede contagiar a otro.

h. El modelo (Figura 4) supone además que los individuos de todas las clases están mezclados y entran en contacto (excepto los que están aislados en hospitales y los muertos). De ahí la importancia de la cuarentena.

Figura 4. Sistema de Ecuaciones del Modelo Clásico SIR

$$
\frac{d S}{d t}=-a I S, \quad \frac{d I}{d t}=a I S-b I, \quad \frac{d R}{d t}=b I,
$$

Aquí $a>0$ es el factor de proporcionalidad de la tasa de contagio y $b$ es la tasa de recuperados por unidad de tiempo.

Por la cuarentena y las diferentes medidas tomadas, la tasa de contagio puede ser reducida (de hecho es el objetivo fundamental al reducir la movilidad social), los datos usados en la modelación son los siguientes: 12 los días de incubación, 2.5 el número básico de reproducción, $\mathbf{R}_{\mathbf{0}}$ (1,5 o menos en cuarentena, o sea, la cantidad de individuos que uno infectado contagia en el período infeccioso), $14 \%$ es la tasa de infección, de esos infectados un 95\% es la 
tasa de recuperados y $5 \%$ es la tasa de mortalidad.

Los Graficos 1, 2, 3 y 4, presentados a continuación, representan una simulación de la evolución de la pandemia en la Ciudad de Corrientes y la Provincia homónima, con una población aproximada de 399999 susceptibles y 1 infectados (999999 para la provincia).

\section{Gráfico 1. Evolución Corrientes-Ciudad}

\section{EvoluciónCtesCiudad}

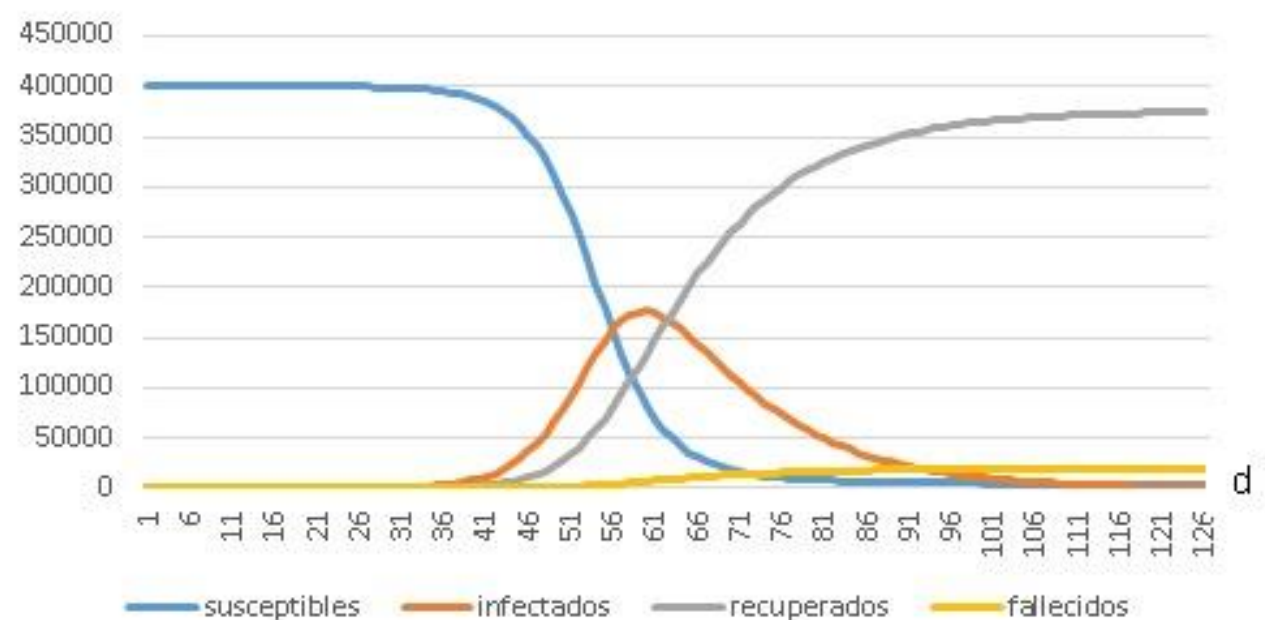

Cuando en la prensa, o en distintos medios, hablan del "pico" de la epidemia, hablan del valor máximo de infectados en un momento dado, así en el Gráfico 1 para la Ciudad de Corrientes y en el Gráfico 2 para la Provincia de Corrientes, el pico se alcanzaría a los 60 días del primer caso reportado, o sea, alrededor del 20 de mayo, siempre que tomemos $\mathbf{R}_{\mathbf{0}}=2,5$ o sea, prácticamente con una vida normal y tendríamos alrededor de 175 mil infectados en la Ciudad y unos 430 mil en la Provincia. Es fácil imaginarse que con esos números todos los servicios públicos hubieran colapsados. En los Gráficos 3 y 4, se presenta la evolución de la epidemia para una tasa mucho menor y se ve fácilmente que el pico máximo se alcanza para la Ciudad casi en el doble del período del tiempo y con 75 mil infectados menos, para la Provincia el pico se alcanza en el doble del período de la Gráfica 2 y se disminuyen en 200 mil los infectados.

Ese es el quid de la cuestión a transmitirle a los estudiantes, guardar la distancia social, cumplir con al cuarentena, es vital para achatar el pico y aplanar la curva, de tal forma que el sistema de salud y otros servicios públicos, puedan asimilar de una manera gradual, las atenciones que requiere la población. 


\section{Gráfico 2. Evolución Corrientes - Provincia}

\section{EvoluciónCtesProv}

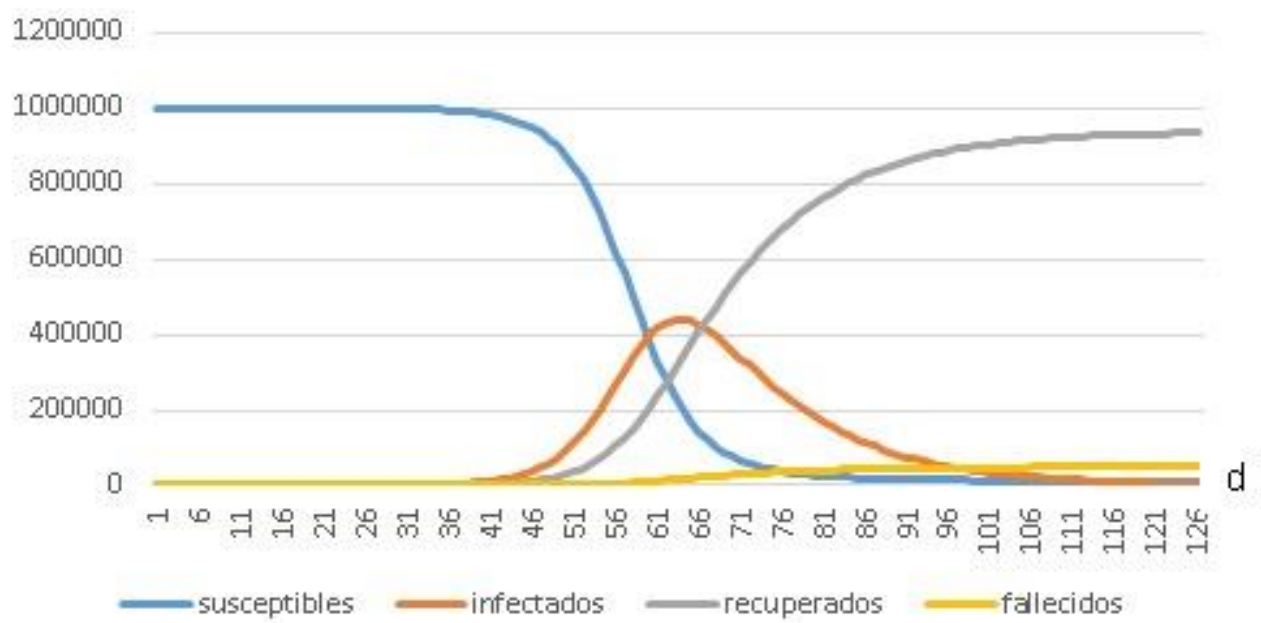

Si consideramos una razón de crecimiento $\left(\mathbf{R}_{\mathbf{0}}\right)$ de 1,5, el comportamiento es el mostrado en el Gráfico 3.

\section{Gráfico 3. Evolución Corrientes - Ciudad}

\section{EvoluciónCtesCiudad}

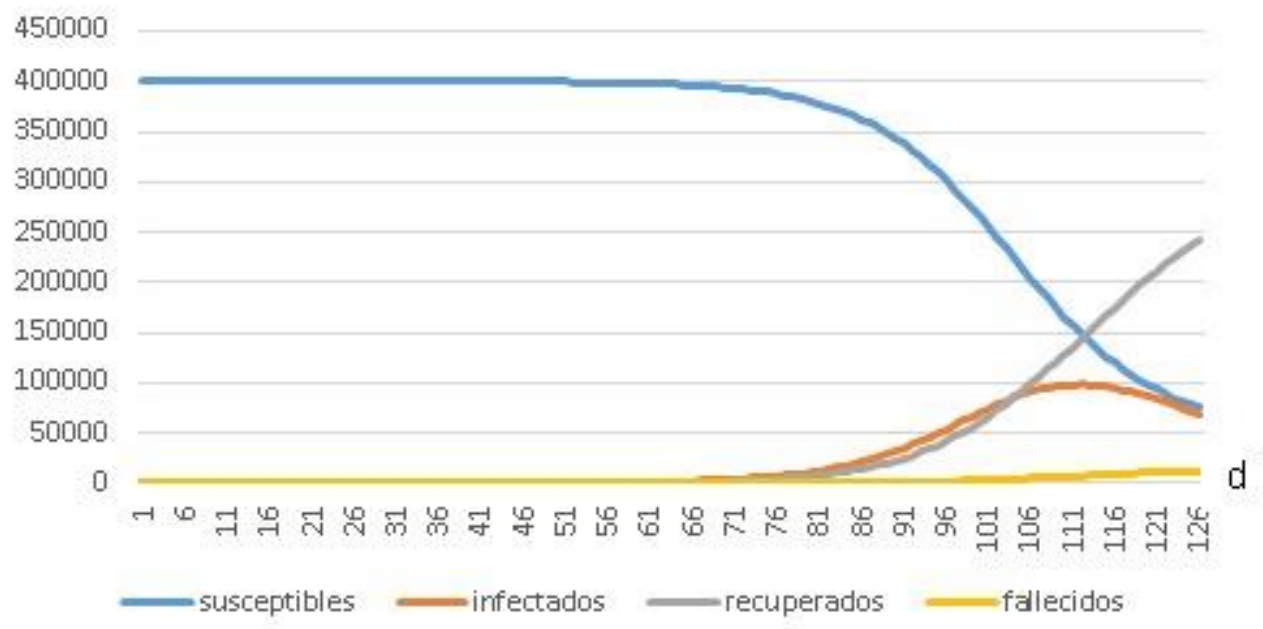




\section{Gráfico 4. Evolución Corrientes - Provincia}

\section{EvoluciónCtesProv}

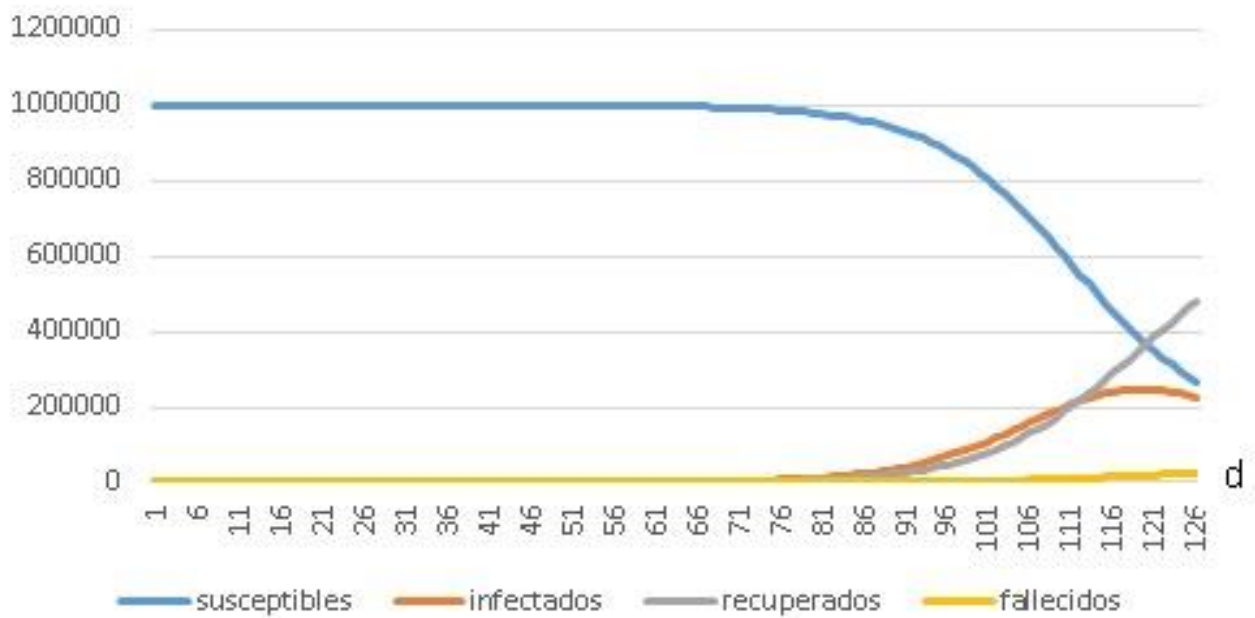

\section{Conclusiones.}

Hemos visto como la Matemática Realista (Heuvel-Panhuizen, 2002), en el proceso de enseñanza-aprendizaje bajo un enfoque de resolución de problemas, donde se haga uso de herramientas matemáticas (desde software como Matemática, GeoGebra hasta el Excel) y de la visualización matemática propicia la experimentación, búsqueda y exploración del conocimiento sobre ecuaciones diferenciales ordinarias, que a la vez estimule y propicie el desarrollo del pensamiento matemático y redunde en el proceso educativo, en la formación de valores y actitudes.

De esta manera, creemos que este proceso de enseñanza-aprendizaje de la resolución de problemas intramatemáticos o extramatemáticos (con modelos basados en ecuaciones diferenciales ordinarias) se perfecciona y se enriquece con los aportes que brinda la integración de la modelación, las TIC, la visualización matemática y la Educación Matemática Realista.

La propuesta que hemos presentado, sin ánimo de completitud, puede servir de ilustración para otros docentes en diferentes áreas, y permite imbricar, de manera natural, desde el conocimiento matemático hasta la formación en valores de los estudiantes.

\section{Referencias}

Arcavi, A. (2003). The role of visual representations in the learning of mathematics. Educational Studies in Mathematics, 52: 215-241. Kluwer Academic Publishers. Printed in the Netherlands. 
De Guzmán, M. (1996). El Rincón de la Pizarra. Cap. 0, el papel de la visualización. Pirámide, Madrid.

Díaz, G. J. y Batanero, M. C. (1994). "Significado institucional y personal de los objetos matemáticos”. En: Recherches en Didactique des Mathématiques. Vol.14, No. 3. pp. 325355.

Freudenthal, H. (1973). Mathematics as an educational task. Dordrecht,Reidel Publishing Co.

Goffree, F. (1993). HF: Working on Mathematics. Education.Educational Studies in Mathematics 25, 21-48, The Legacy of Hans Freudenthal. Kluwer Academic Publishers. Holanda.

Halmos, P. R. (1980). The Heart of mathematics. American mathematical Monthly, 87, 519524.

Hershkowitz, R. (1990). Psychological aspects of learning geometry. En P. Nesher y J. Kilpatrick (Eds.), Mathematics and cognition (pp. 70-95). Cambridge, G.B.: Cambridge U. P.

Jourdain, P.E.B. (1976). La Naturaleza de la Matemática. En James R. Newman (comp.). "Sigma. El Mundo de la Matemática", Ediciones Grijalbo, Barcelona-Buenos AiresMéxico, 1976, tomo 1, 343-408.

Kermack, W. O. and McKendrick, A.G. (1927), Contributions to the Mathematical Theory of Epidemics, Proc. Roy. Soc. A. vol. 115, 700-721.

Nápoles V., J. E. (1997). "Sobre el significado de los objetos matemáticos. El caso de los irracionales". En: Memorias COMAT'97. Cuba: Universidad de Matanzas.

Santos, M., Moreno, L. \& Camacho, M. (2016). Problem solving and the use of digital technologies within the Mathematical Working Space framework. ZDM 48 (6), p. 828.

Uribarri, S. G.. Rodríguez Meza, M. A. y Cervantes Cota, J. L. Las matemáticas de las epidemias: caso México 2009 y otros, CIENCIA ergo-sum, Vol. 20-3, noviembre 2013febrero 2014. Universidad Autónoma del Estado de México, Toluca, México. Pp. 238-246.

Van den Heuvel-Panhuizen, M. (2002). Towards scientific research in classrooms. Researching mathematics classrooms: a critical examination of methodology (London, Praeger), 111115.

Zimmermann, W. \& Cunningham, S. (1991). What Is Mathematical Visualization? Visualization in Teaching and Learning Mathematics. Eds. MAA Notes Number 19, 1991. 